\title{
Successful on-pump surgery for treatment of a life threatening cardiopulmonary oncologic mass
}

Sébastien Queron ${ }^{1}$, Patrick Van Ruyssevelt ${ }^{2}$, Marc Beauduin ${ }^{3}$, Marie-Odile Penny ${ }^{4}$, Luc Van Obbergh ${ }^{5}$, Servais $\mathbf{M}^{2}$, Edo wijtenburg${ }^{2}$ and Véry Coulic ${ }^{6}$

${ }^{1}$ Cardio-vascular Surgery Department, Clinique Saint Luc, Bouges, Brussels, Belgium

${ }^{2}$ Cardiovascular and Thoracic Surgery, Jolimont Hospital, Haine-Saint-Paul, Brussels, Belgium

${ }^{3}$ Pneumology and Oncology, Jolimont Hospital, Haine-Saint-Paul, Brussels, Belgium

${ }^{4}$ Anatomopathology, Jolimont Hospital, Haine-Saint-Paul, Brussels, Belgium

${ }^{5}$ Anesthesiology Department, CHU Erasme, Jolimont Hospital, Haine-Saint-Paul, Brussels, Belgium

${ }^{6}$ Laboratory of Experimental Medicine, Université Libre de Bruxelles, Centre Hospitalier Universitaire, Brugmann site Horta, Brussels, Belgium

\begin{abstract}
The case of a 39 years old woman with a cardiopulmonary oncologic mass successfully treated by complete resection is reported. In 1998 she underwent resection of the mandible for a grade II mesenchymal chondrosarcoma. In 2009, a new central lesion in the left lung extending to the left atrium wall was discovered. No other metastasis was found at extensive screening. The immediate life-threatening situation due to dyspnea required surgical intervention without delay. The complete resection of a malignant tumor under cardiopulmonary bypass is discussed. An isolated adrenal metastasis was successfully surgically treated in 2013 . October 2015 , the patient has no evidence of disease.
\end{abstract}

\section{Introduction}

Mesenchymal chondrosarcoma is a rare very aggressive highly malignant tumor characterized by a biphasic appearance [1]. Mesenchymal chondrosarcoma is a rare histological variant of chondrosarcoma (represents 3 to $10 \%$ of all chondrosarcomas), very aggressive, characterized by a biphasic histological appearance with on one side a chondrosarcomatous aspect, well differentiated (bands of cartilage matrix), and on the other one very small round cell, undifferentiated. This includes a very rich blood supply, and then the differential diagnosis with hemangiopericytoma can be discussed.

This lesion has a peak frequency is located between $2^{\text {nd }}$ and $3^{\text {rd }}$ decades of life. The impact between the two sexes is identical. Most often they are tumours with very high metastatic potential. Oncogenic osteomalacia has been reported in connection with this tumour.

Mesenchymal chondrosarcomas have preferential anatomical sites, including the flat bones (pelvis, ribs, face bones), long bones are rarely affected.

It is a very high malignant potential tumour type with a tendency to local recurrence and distant metastases, sometimes observed even after a delay of more than 20 years.

The clinical course of this type of injury is often very long, which absolutely requires a long-term oncological follow.

It should be noted that the mesenchymal chondrosarcoma of the jaw seems to develop itself more slowly than in other anatomical sites.

The concept of oncological surgery combined to a cardiopulmonary bypass for long has been considered as unsuitable because of the risk of tumor cell dissemination [2].

\section{Report of the case}

In 2009 a 39 years old woman was admitted at Jolimont hospital for assessment of a left basal infective infectious pneumonitis. Rapidly a left central pulmonary mass involving the left atrial wall was discovered. CT scan and echography have demonstrated a huge intraluminal growth of the tumor nearly trough the mitral valve (Figure 1):

Collected anamnesis has shown that in the year 1997the patient underwent a resection of the left jaw for a grade II mesenchymal chondrosarcoma. Two-step reconstruction with autologous fibula (1998) and iliac crest bone withlatissimus dorsi muscle (2001) was performed, Radio and chemotherapy (Adriamycine, Ifosfamide, uromitexan) were performed. A close yearly follow up control was negative till the current event

A surgicalbiopsy of the left central pulmonary mass made by a short left mini-thoracotomy revealed a late metastasis of the original jaw's tumor.

Because of the cardiologic threat and the oncologic situation (+ $\mathrm{XX} \%$ within 2 months), taking also into account the young age of the

Correspondence to: Sebastien Queron, M.D., Department of Cardio-vascular Surgery-Clinique saint Luc, Rue saint Luc, $\mathrm{N}^{\circ} 8$, Bouges, Belgium, Tel:+ 32.0477.13.73.473; E-mail: sebastien.queron@slbo.be

Véry Coulic, Laboratory of Experimental Medicine, Université Libre de Bruxelles, Centre Hospitalier Universitaire, Brugmann site Horta, Brussels, Belgium, E-mail: coulic.very@belgacom.net; very.coulic@chu-brugmann.be

Received: October 29, 2015; Accepted: November 26, 2015; Published: November 30, 2015 
a).

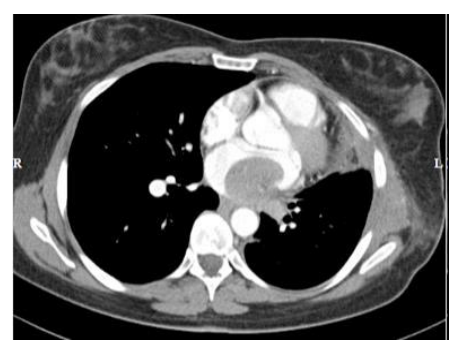

b)

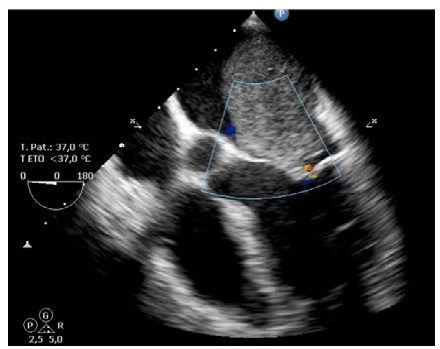

c)

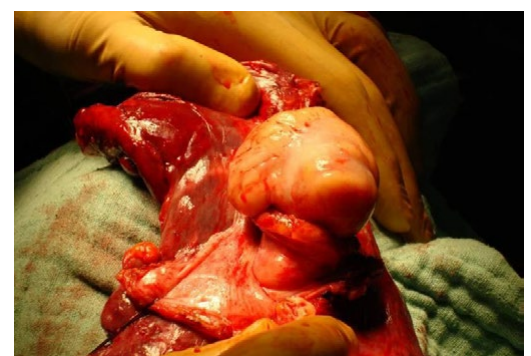

Figure 1. Pre operation views: tumour mass.

a) CT scan image

b) Ultrasound image

c) Operation piece

patient, a radical treatment was considered. The multidisciplinary medical team, regardless of disseminating neoplastic cells risks, decided rapid and radical surgical treatment.

On the 06/30/2009a classic posterior lateral thoracotomy through the fifth left intercostal space was performed. Through a left groin approach, the femoral vessels were also prepared. Because of the tight connection between tumorous and surrounding tissues, removal of the left lung was required for a complete extirpation of the tumor. Extemporaneous histological investigation of the aortic lymph nodes was negative. After division of the pulmonary artery and the main bronchus, a normothermic heparin coated cardiopulmonary bypass via the left femoral vessels (heparin $2 \mathrm{mg} / \mathrm{kg}$ for a target ACT200) was started. After aortic cross clamping and antegrade blood cardioplegia (Calafiore's protocol), the left atrium was widely opened around the pulmonary veins, allowing a R0 resection of a $5 \times 5 \mathrm{~cm}$ intra cardiac mass together with the entire left lung (Figure 2a). Left atrium reconstruction was performed using a $16 \mathrm{~cm}^{2}$ equine pericardial patch with a 4.0 polypropylene running suture. After venting, the aorta clamp wasreleased 52 minutes later and the patient was easily weaned after a $\mathrm{CPB}$ time of 83 minutes. No mitral dysfunction was observed at the echocardiography).

\section{The ICU stay lasted $48 \mathrm{~h}$ and was uneventful.}

Histological diagnosis was: mesenchymal chondrosarcoma Grade II, absence of lymph node metastasis in all the lymph nodes removed (peri-hilum, left pulmonary artery and the aorto-pulmonary window). The bronchial section slice, as well as the auricular margin was pathology free.

In this case, the team's pathology laboratory has for a long time believed in the diagnosis of a recurrence of a hemangiopericytoma. The tumour formation was characterized by alternating areas of cartilage with varying degrees of maturation, sometimes calcified, and areas

of round cells, associated with abundant vascularization; cells of the hemangiopericyte type were monotonous. The cytoplasm was not abundant; nuclei were ovoid with fine nucleoli. The mitotic activity was relatively low. Finally, the diagnosis of mesenchymal chondrosarcoma Grade II was adopted.

After an uneventful post operation course, the patient was discharged at day 10 .

Three months after surgery, CT scan has shown a complete filling of the left thoracic cavity; at the echocardiography control no hemodynamic abnormalities were found (Figures $2 \mathrm{~b}$ and $2 \mathrm{c}$ ). The patient remained under very regular monitoring of the oncologic team.

In January 2013, an isolated adrenal metastasis was diagnosed and removed by laparoscopic surgery.

In July 2015, the patient had no evidence of disease: no specific complain, stable weight, negative clinical examination. The last biological control showed normal range values. At control thoracic CT-Scan, no suspect lesion or lymphadenopathy in the lung could be identified. PET-FDG whole body scans were negative.

a)

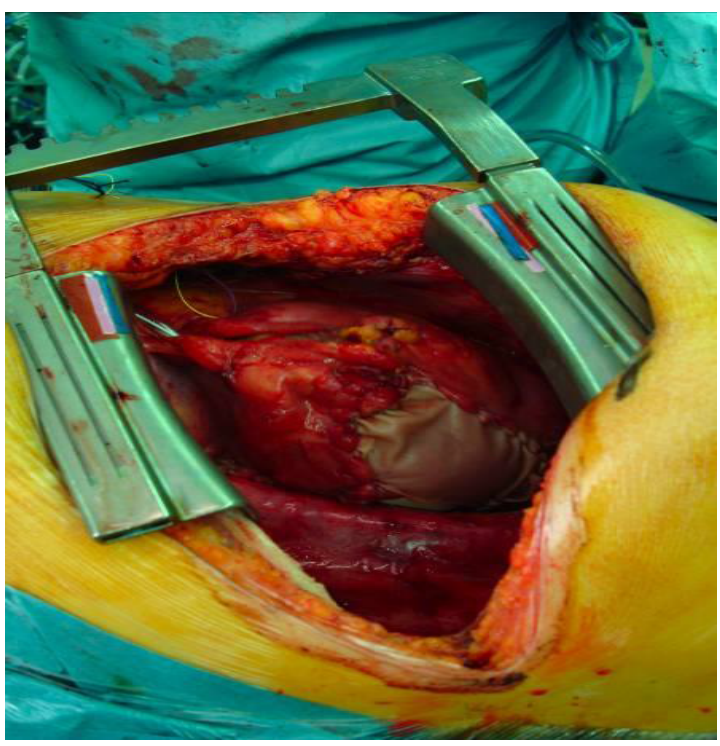

b)

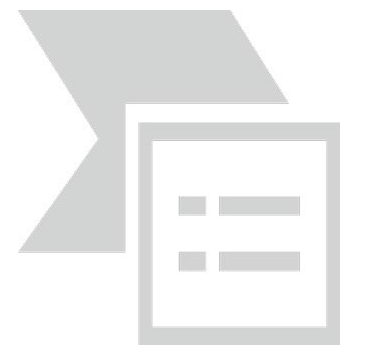

Figure 2. Post operation views: tumour mass.

a) Operation site

b) CT scan image

c) Ultra sound image c)

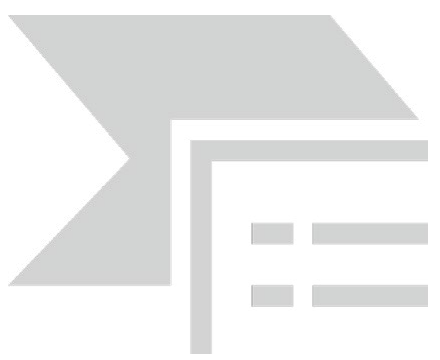




\section{Discussion}

In this very case, among different options considered, radiation therapy or chemotherapy were excluded because of the cardiologic threat and the insufficient information about tumor histology. The surgical option seemed to be acceptable and allowed a radical excision. The concept of oncological surgery in combination with a cardiopulmonary bypass has been for a long time considered as unacceptable due to the theoretical risk of malignant cells dissemination $[1,2]$.

Some publications have demonstrated the feasibility and the reliability of the association of oncologic surgery not only with thoracic,but also with digestive and urological surgery [2]. It seems that these patients do not present more complications after combined surgery, no significant higher rate of malignancy recurrence at short and long-term follow-up. Suzuki et al. [3] has identified 74 patients with neoplastic disease for whom cardiac surgery was considered. After a $42 \pm 37$ months follow-up, it was not possible to demonstrate significant differences in terms of malignancy recurrence among patients undergoing on or off bypass cardiac surgery. The authors also found no difference, neither in mortality, related to neoplastic disease nor in terms of 2 and 5 years survival after on-pump or off-pump cardiac surgery. Finally, it is possible to conclude that cardiopulmonary bypass can be used in cancer patients who require cardiac surgery.

Another study [4] performed in patients requiring cancer and cardiac surgery with cardiopulmonary bypass has shown an excellent immediate post-operative course, and 100\% survival one year after surgery. Cytological investigations after each operation could detect tumor cells only at the surface of the cardiopulmonary bypass arterial filter circuit ( $20 \mu$ pores). It is possible to conclude that with the right equipment, performing cardiac surgery with extracorporeal circulation in oncological situations without increasing the risk of neoplastic cell dissemination is possible.

\section{Conclusions}

The interest of this case was to show the feasibility of a difficult oncological surgery in a particularly difficult anatomical access, by a combined cardio-thoracic surgical team, on a young patient whose prognosis was compromised at short term.

R0 oncological surgery, under cardiopulmonary bypass, has been performed in semi-emergency and has not only been able to precise the histological diagnosis of the lesion, but also has saved the life of this young patient who is still in complete remission 6 years after main surgery.

\section{References}

1. DeVitaVTJr, Lawrence ThS, Rosenberg SA (2012) Cancer: Principles \& Practice of Oncology, 9th edition.

2. Dedeilias P, Koletsis E, Rousakis AG, Kouerinis I, Zaragkas S, et al. (2009) Deep hypothermia and circulatory arrest in the surgical management of renal tumors with cavoatrial extension. J Card Surg 24: 617-623. [Crossref]

3. Suzuki S, Usui A, Yoshida K, Matsuura A, Ichihara T, et al. (2010) Effect of cardiopulmonary Bypass on cancer Prognosis. Asian Cardiovasc Thorac Ann 18: 536540. [Crossref]

4. Akchurin RS, Davidov MI, Partigulov SA, Brand JB, Shiriaev AA, et al. (1997) Cardioplumonary bypass and cell-saver technique in combined oncologic and cardiovascular surgery. Artif Organs 21: 763-765. [Crossref]

Copyright: (C2015 Queron S. This is an open-access article distributed under the terms of the Creative Commons Attribution License, which permits unrestricted use, distribution, and reproduction in any medium, provided the original author and source are credited. 